Tri-Linh C. Lu

Christoph H. Huber

Elena Rizzo

Jashmid Dehmeshki

Ludwig K. von Segesser

Salah D. Qanadli

\section{Ascending aorta measurements as assessed by ECG-gated multi-detector computed tomography: a pilot study to establish normative values for transcatheter therapies}

Received: 27 April 2008

Revised: 15 July 2008

Accepted: 31 July 2008

Published online: 23 September 2008

(C) European Society of Radiology 2008
There is no conflict of interest or financial support concerning this study.

T.-L. C. Lu · E. Rizzo $\cdot$ S. D. Qanadli

Department of Radiology, University

Hospital Lausanne (CHUV),

Lausanne, Switzerland

C. H. Huber - L. K. von Segesser

Department of Cardiovascular Surgery,

University Hospital Lausanne (CHUV),

Lausanne, Switzerland

\section{J. Dehmeshki}

Digital Imaging Research Centre,

Faculty of Computing,

Information Systems and Mathematics,

Kingston University,

London, UK
S. D. Qanadli $(\bowtie)$

Service de Radiodiagnostic et de

Radiologie interventionnelle, BH-07,

Centre Hospitalier Universitaire

Vaudois,

Rue du Bugnon 46,

1011 Lausanne, Switzerland

e-mail: salah.qanadli@chuv.ch

Tel.: +41-21-3144560

Fax: +41-21-3144488

\begin{abstract}
The aim of this study was to provide an insight into normative values of the ascending aorta in regards to novel endovascular procedures using ECG-gated multi-detector CT angiography. Seventy-seven adult patients without ascending aortic abnormalities were evaluated. Measurements at relevant levels of the aortic root and ascending aorta were obtained. Diameter variations of the ascending aorta during cardiac cycle were also considered. Mean diameters ( $\mathrm{mm}$ ) were as follows: LV outflow tract $20.3 \pm 3.4$, coronary sinus $34.2 \pm 4.1$, sino-tubular junction $29.7 \pm 3.4$ and mid ascending aorta $32.7 \pm 3.8$ with coefficients of variation $(\mathrm{CV})$ ranging from 12 to $17 \%$. Mean distances $(\mathrm{mm})$ were: from the plane passing through the proximal
\end{abstract}

insertions of the aortic valve cusps to the right brachio-cephalic artery (BCA) $92.6 \pm 11.8$, from the plane passing through the proximal insertions of the aortic valve cusps to the proximal coronary ostium $12.1 \pm 3.7$, and between both coronary ostia $7.2 \pm 3.1$, minimal arc of the ascending aorta from left coronary ostium to right BCA 52.9 \pm 9.5 , and the fibrous continuity between the aortic valve and the anterior leaflet of the mitral valve $14.6 \pm 3.3, \mathrm{CV}$ $13-43 \%$. Mean aortic valve area was $582.0 \pm 131.9 \mathrm{~mm}^{2}$. The variation of the antero-posterior and transverse diameters of the ascending aorta during the cardiac cycle were $8.4 \%$ and $7.3 \%$, respectively. Results showed large inter-individual variations in diameters and distances but with limited intra-individual variations during the cardiac cycle. A personalized approach for planning endovascular devices must be considered.

Keywords ECG-gated computed tomography - Normative values · Ascending aorta $\cdot$ Endovascular repair - Endograft

\section{Introduction}

Historically treatment of abdominal aortic aneurysms (AAA) was based on surgical repair until the 1990s [13]. Since then endovascular graft systems have been widely recognized and used for AAA repair (EVAR) [4]. The lack of off-the-shelf endografts warrants precise prepoperative imaging to define adequate device selection. Therefore increasing importance has been placed on preoperative CT imaging protocols for the planning of endovascular abdominal aortic aneurysms [5]. With the advent of endovascular aneurysm repair and valve replacement, 
more focus is now turned to ascending aorta and aortic arch problems $[6,7]$. Experience with endografts for ascending aortic disease is scarce because few groups are using such devices due to technological restrictions. On the one hand the lower caseload for ascending aorta endoluminal procedures limits the device evolution. On the other hand the large diameter and the curvature of ascending aorta and aortic arch render access to these vascular structures difficult and hinder the introduction, placement, tracking and deployment of the graft, thus challenging device engineering. Blood flow in the coronary arteries and the supra-aortic arteries should also be maintained, by means of different fenestrated technologies and surgical bypasses depending on the graft landing zone $[8,9]$.

All these anatomical and technical constraints require precise knowledge of normal ascending aorta. Although normative thoracic aortic dimensions have already been determined by means of CT angiography [10-13], to our knowledge, no exhaustive measurements have been established by means of ECG-gated CT angiography.

Despite improvement in spatial and temporal resolution (below $150 \mathrm{~ms}$ ) with fast-rotating multi-detector CT technology (MDCT), the thoracic aorta remains a vascular segment that is difficult to analyse due to axial and transverse motion during the cardiac cycle, which is particularly true for the ascending aorta [14-16]. ECG assistance for aortic studies allows the synchronisation of data acquisition to the heart cycle in order to obtain CT data during the diastolic phase when heart movement is minimal thus reducing motion artefact [17-19].

The purpose of this study was to provide an insight into normative values of the ascending aorta and its branches in adults, regarding endovascular procedures and devices by using ECG-gated MDCT.

\section{Methods}

\section{Study population}

Seventy-seven patients were included in this study. There were 59 men and 18 women. Age ranged from 22 to 83 years. Mean age and median were 54.7 and 54 years, respectively. Inclusion criteria were: patients older than 18 years old evaluated for coronary artery disease with data available from the outflow tract up to the mid ascending aorta or up to the right brachio-cephalic artery. Exclusion criteria were: outflow tract and/or aortic valve diseases, atherosclerosis of the ascending aorta, dissection or aneurysm of the ascending aorta, surgical procedures of the ascending aorta, congenital anomalies of the ascending aorta, important artefacts. Patients who were not under beta-blocker therapy received oral beta-blocker before examination and mean heart rate during acquisition was $68.9 \mathrm{bpm}$.

\section{CT protocol}

Data were acquired on a 64-detector VCT system (General Electric Medical Systems, Milwaukee, USA). Acquisitions were obtained after an antecubital intravenous injection of 100-120 ml nonionic contrast medium (Accupaque 350, GE Healthcare, Giles, UK) with $40 \mathrm{ml}$ saline solution as bolus chaser at $4-5 \mathrm{ml} \mathrm{s}^{-1}$. Helical $\mathrm{CT}$ acquisition was started during a single breath hold when sufficient contrast enhancement (at least $200 \mathrm{HU}$ ) was detected in the ascending aorta (bolus tracking technique). All CT data were obtained with a collimation of $0.625 \times 40 \mathrm{~mm}$, a FOV of $350 \mathrm{~mm}$, rotation time of $0.35 \mathrm{~s}$ and $120 \mathrm{kV}$ with $\mathrm{mA}$ modulation during the cardiac cycle (typically 250$750 \mathrm{~mA})$. High resolution $(0.625-\mathrm{mm}$ slice thickness, $512 \times 512$ matrix) retrospective ECG-gated axial transverse reconstructions were obtained for all patients at $75 \%$ of the $\mathrm{R}-\mathrm{R}$ cycle. Multi-phase reconstructions were also done from 0 to $99 \%$ with increments of 6 or $10 \%$.

\section{Data analysis and aortic measurements}

In 49 patients, data were available from the outflow tract up to the mid ascending aorta. Data were available up to the right brachio-cephalic artery in the remaining patients $(n=28)$.

Analysis of ascending aorta and aortic arch morphology was performed on a Advantage Window workstation with version 4.3 software (GE Medical Systems). Twodimensional multiplanar reformations and automatic, interactive, centerline-based segmentation of the aorta were used and reviewed in consensus by two radiologists. The reported precision of measurements of the software is less than $0.7 \mathrm{~mm}$.

In close collaboration with cardiovascular surgeons, relevant measurements (Fig. 1) were identified as key parameters for development of future transcatheter cardiovascular therapies (TCT): aortic diameters perpendicular to the aortic centerline axis (Fig. 2) at different levels (outflow tract measured at the level of the insertion of the anterior leaflet of the mitral valve, coronary sinus, sino-tubular junction and mid ascending aorta level); distances from the plane passing through the proximal insertions of the aortic valve cusps in the sinus wall to the right brachio-cephalic artery, from the plane passing through the proximal insertions of the aortic valve cusps to the proximal coronary ostium, between both coronary ostia; and the minimal arc of the ascending aorta from the left coronary ostium to the level of the right brachio-cephalic artery. Aortic valve area was also measured.

Dynamic analysis was done for measurement of the length of the aorto-mitral fibrous continuity in every patient. Anatomically it refers to the septum extending from below the non-coronary and the left coronary cusps to the insertion of the anterior leaflet of the mitral valve [20]. 


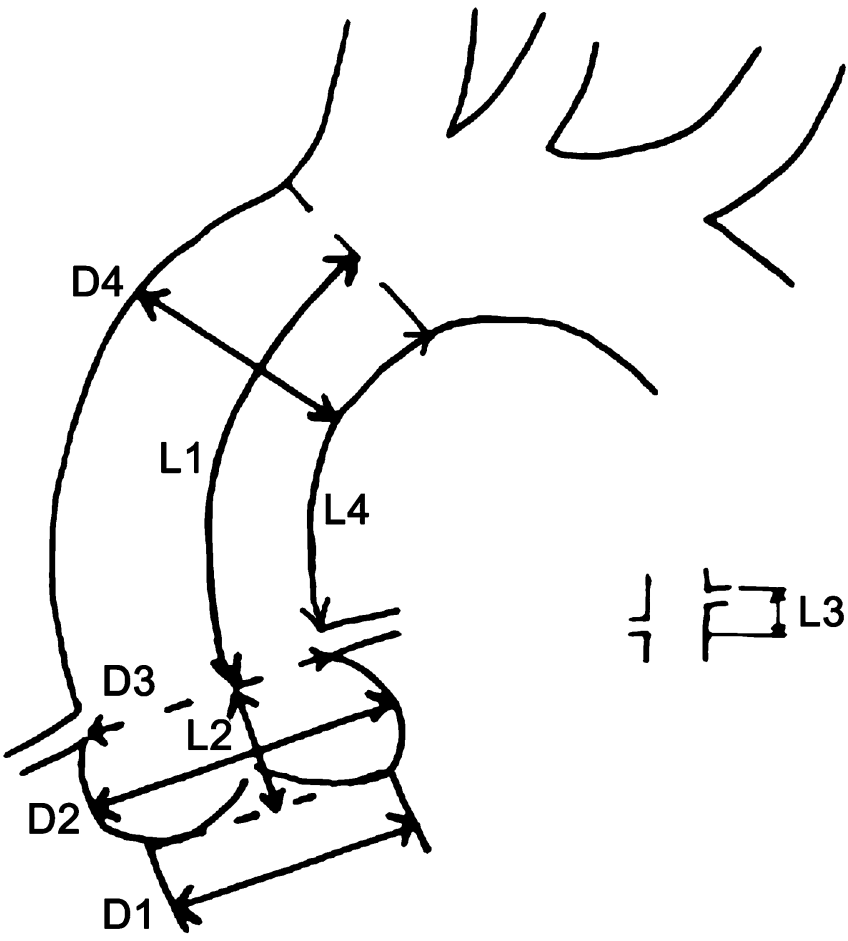

Fig. $1 D 1$ outflow tract, D2 coronary sinus, D3 sino-tubular junction, D4 mid ascending aorta, L1-L2 distance from the plane passing through the proximal insertions of the aortic cusps to the right brachio-cephalic artery, L2 distance from the plane passing through the proximal insertions of the aortic cusps to the proximal coronary ostia, $L 3$ distance between both coronary ostia, $L 4$ minimal arc of the ascending aorta from the left coronary ostium to the right brachio-cephalic artery

This septum was deemed to be a potential site for graft anchoring. It was identified on a three-cavities view (Fig. 3) during the cardiac cycle and its length was measured from the proximal insertions of the left coronary and the non-coronary cusps in the sinus wall

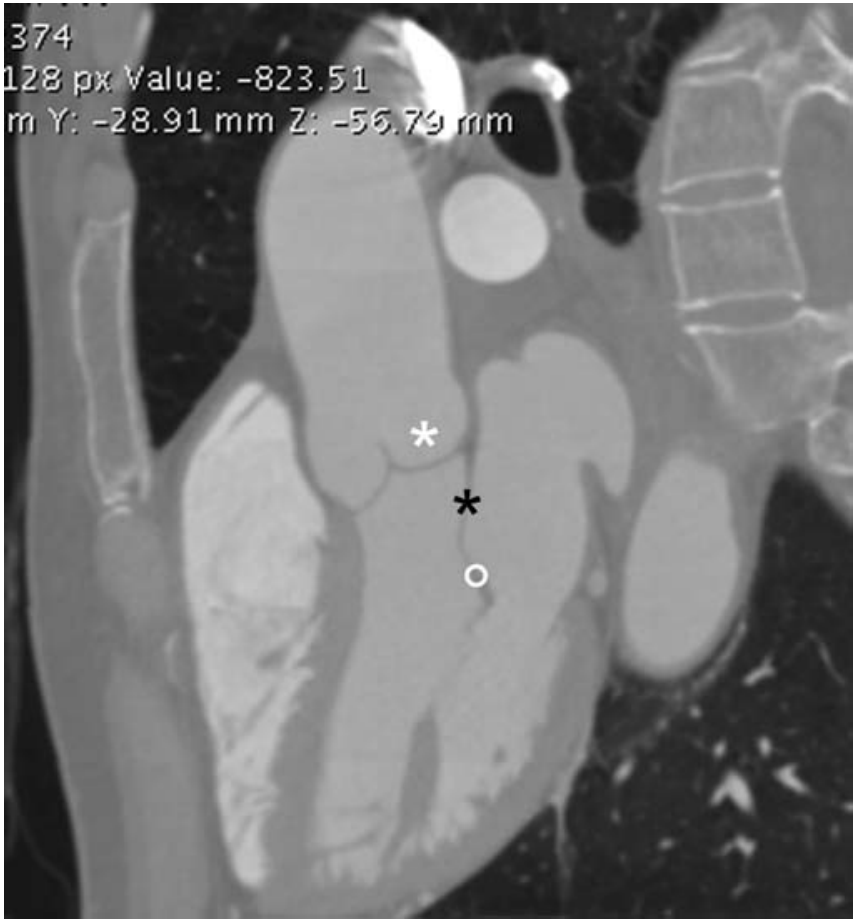

Fig. 3 Three-cavities view demonstrates the fibrous continuity (black asterisk) extending from the left coronary cusp (white asterisk) to the insertion of the anterior leaflet (white circle) of the mitral valve

to the insertion of the anterior leaflet of the mitral valve. Dynamic data were also used for measurement of the ascending aorta pulsatility. Pulsatility was defined as the percentage difference of minimal and maximal anteroposterior and latero-medial diameters of the mid ascending aorta.

Mean, median, maximum and minimum values were considered with standard deviations. Coefficients of variation were also computed.
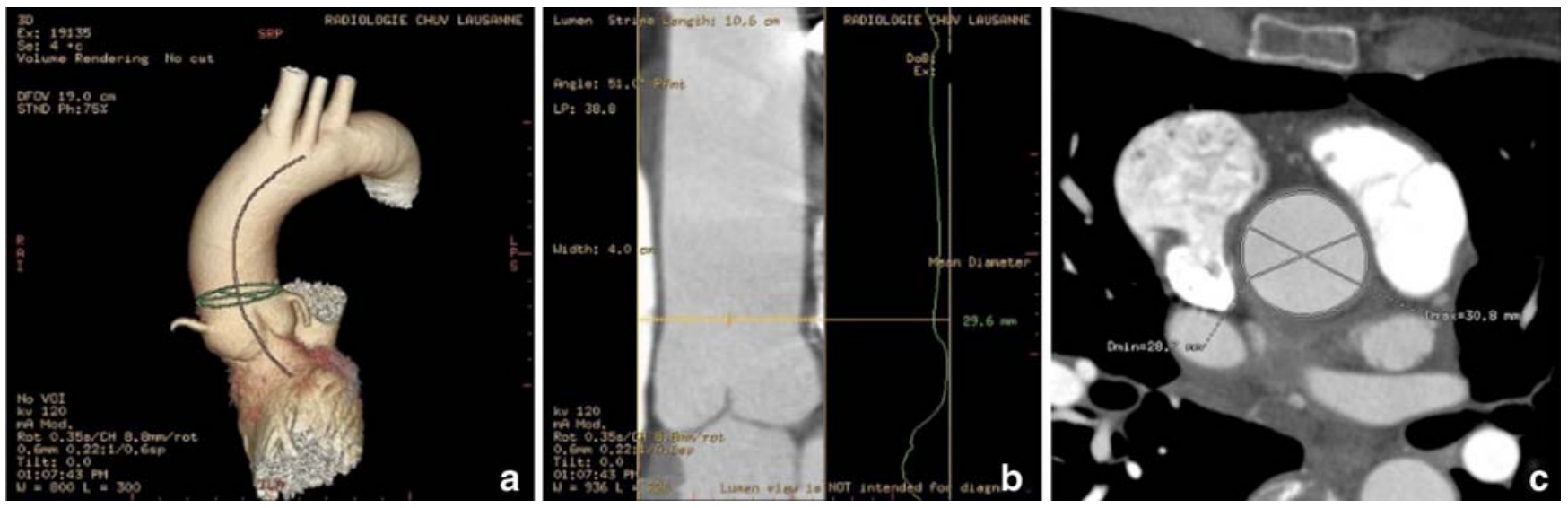

Fig. 2 Example of measurement at the level of the sino-tubular junction in a 54-year-old woman. Volume rendering reconstruction of the ascending aorta and of the aortic arch (a). Lumen view of the ascending aorta (b). Automatic segmentation of the ascending aorta (c) 


\section{Results}

Mean diameters were respectively $20.3 \pm 3.4 \mathrm{~mm}$ for outflow tract, $34.2 \pm 4.1 \mathrm{~mm}$ for coronary sinus, $29.7 \pm$ $3.4 \mathrm{~mm}$ for sino-tubular junction and $32.7 \pm 3.8 \mathrm{~mm}$ for mid ascending aorta. Coefficients of variation ranged from 12 to $17 \%$. See Table 1 for details. The stratification of diameters by age is presented in Fig. 4.

Mean distance from the plane passing through the proximal insertions of the aortic valve cusps to the right brachio-cephalic artery was $92.6 \pm 11.8 \mathrm{~mm}$. Mean distance from the plane passing through the proximal insertions of the aortic valve cusps to the proximal coronary artery was $12.1 \pm 3.7 \mathrm{~mm}$ with a coefficient of variation of $31 \%$. Mean distance between both coronary ostia was $7.2 \pm 3.1 \mathrm{~mm}$ with a coefficient of variation of $43 \%$. Mean value of the minimal arc of the ascending aorta from the left coronary ostium to the level of the right brachio-cephalic artery was $52.9 \pm 9.5 \mathrm{~mm}$. Mean value for the aorto-mitral fibrous continuity was $14.6 \pm$ $3.3 \mathrm{~mm}$ with a coefficient of variation of $23 \%$.

Antero-posterior and transverse diameters of the mid ascending aorta during the cardiac cycle varied from a maximum of $34.2 \mathrm{~mm}$ to a minimum of $31.3 \mathrm{~mm}$ and from a maximum of $33.6 \mathrm{~mm}$ to a minimum of $31.2 \mathrm{~mm}$, respectively. Computed variations are respectively $8.4 \%$ and $7.3 \%$.

Mean area of the aortic valve was $582.0 \pm 131.9 \mathrm{~mm}^{2}$ with a coefficient of variation of $23 \%$. The median value was 579.1. The maximum and minimum values were 971.0 and $286.5 \mathrm{~mm}^{2}$, respectively.

\section{Discussion}

Transcatheter therapies of the aortic valve and of the ascending aorta will bring an innovative approach to the treatment of thoracic aorta abnormalities. Nevertheless indications for such types of minimal invasive procedure are still limited. The main restrictions are due to the size limitation of the vascular access and the availability of

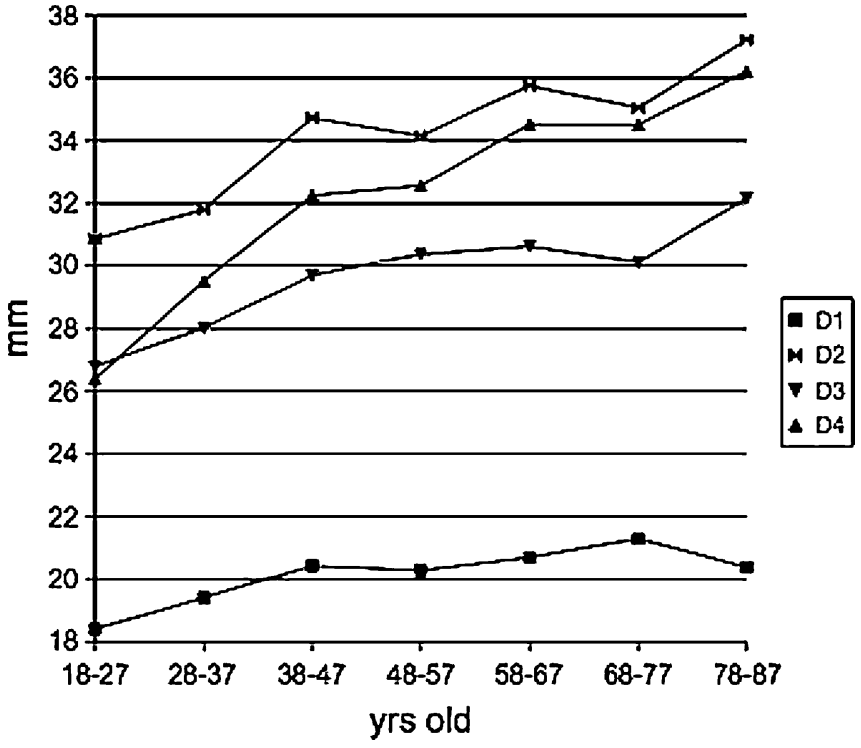

Fig. 4 Mean aortic diameters by age. $D 1$ outflow tract, $D 2$ coronary sinus, D3 sino-tubular junction, D4 mid ascending aorta

dedicated devices. The latter are now being developed, but in the pursuit of the ideal ascending aorta endograft precise morphometric measurements of this vascular segment are needed. This study presents an insight into measurements of the ascending aorta by means of ECG-gated tomography.

ECG-gated MDCT provides high resolution images in a near isotropic condition that allows precise 3D measurements. Although MDCT imparts a high dose of ionizing radiation [21], it remains the gold standard for quantitative imaging owing to its high spatial resolution. MDCT allows fast and readily accessible acquisition of volume data that are prone to easy post-processing. Besides, as already mentioned, motion artefacts - particularly in the ascending aorta - can affect precision of measurements when examination is not ECG-gated.

To our knowledge, no previous studies have reported complete assessment of the ascending aorta by quantifying various parameters. However our results agreed with those

Table 1 Results obtained from ECG-gated multi-detector CT angiography (mm)

\begin{tabular}{lllllll}
\hline & Mean & SD & Median & Minimum & Maximum & CV (\%) \\
\hline D1 & 20.3 & 3.4 & 20.1 & 12.1 & 28.6 & 17 \\
D2 & 34.2 & 4.1 & 34 & 25.1 & 45.4 & 12 \\
D3 & 29.7 & 3.4 & 29.8 & 21.5 & 37.8 & 12 \\
D4 & 32.7 & 3.8 & 32.3 & 23.3 & 42.5 & 12 \\
L1-L2 & 92.6 & 11.8 & 93.7 & 72.3 & 119.9 & 13 \\
L2 & 12.1 & 3.7 & 12.1 & 2.9 & 25.6 & 31 \\
L3 & 7.2 & 3.1 & 6.6 & 2.4 & 17.5 & 43 \\
L4 & 52.9 & 9.5 & 52 & 32.7 & 78 & 18 \\
Fibrous continuity & 14.6 & 3.3 & 14.7 & 9.2 & 26 & 23 \\
\hline
\end{tabular}


reported in previous studies. In two studies with non-gated CT in adult patients, the diameter of the aortic sinus was measured between 29.8 and $36.2 \mathrm{~mm}$ and the diameter of the ascending aorta between 30.9 and $35.1 \mathrm{~mm}[10,13]$. A Dutch group used gated CT to measure the distance between the aortic valve and the right brachio-cephalic in 14 patients [22]. Their results ranged from 72 to $99 \mathrm{~mm}$. This group also showed that motion and stress forces in the ascending aorta are higher than in the abdominal aorta with a maximum difference of diameter during the cardiac cycle of up to $27.5 \%$. Our results differ with this reported variation. Such a difference may be due to the studied populations. Our sample was larger and younger (77 patients versus 15 , mean age 54.7 years versus 72.2 ) and heart rates during examination were perhaps different. Nevertheless based on the age-related reduction of distensibility of the aortic wall [23] we think that pulsatility in the ascending aorta especially in the elder patients is probably not that high.

Several other imaging techniques have already been used for evaluating the ascending aorta. In the pediatric population, morphometric measurements have been done with transthoracic echocardiography [24]. Although transthoracic echocardiography or even transesophageal echocardiography are readily available techniques and although gated-ultrasound technologies offer even better temporal resolution compared with computed tomography [25], image acquisition and interpretation are still operator dependent [26]. Interposition between the ascending aorta and the trachea make visualization difficult and sometimes incomplete [27]. Artefacts can also appear in the ascending portion of the aorta due to reflection of ultrasound on the atrio-aortic interface and can cause false-positive findings for example in diagnosis of aortic dissection [28].

Normative data were also obtained by means of angiographic acquisition in the pediatric population [29, 30]. The technique is based on projectional views and summation images. Thus angle of incidence of X-rays and overlapping structures can induce geometrical distortion [31]. Considering that angiography also requires an invasive procedure, it is not the most suitable technique to get aortic measurements.

Magnetic resonance angiography experience for normative measurements was also described. However few data are available [32]. Cardiac magnetic resonance imaging is a promising technology. Nevertheless reduced availability, cost and patient discomfort are disadvantages compared with tomography. From a technical point of view fast acquisition sequences like echo-planar imaging (EPI) introduce geometric distortion and ghosting in the cardiac examination post-processing and reconstruction [33]. Strong magnetic fields can also interfere with the ECG signal recording during MRI scans, the so-called magnetohydrodynamic effect [34].

Ideally one standard endovascular device should fit any patient's anatomy. Experience with EVAR showed that this is practically impossible and careful selection of size, geometrical configuration and anchoring options is needed, depending on anatomical morphology and the nature of the lesion to be treated [35]. Our results confirmed that large inter-individual variations exist. Distances between coronary ostia and the level of insertions of the aortic valve cusps and mean distances between both ostia showed coefficients of variation up to $43 \%$. Variation of coronary anatomy is common [36] and becomes problematic when a fenestrated endograft is in scope [37]. When results are stratified by age, differences exist with a tendency to have larger aortic diameters with older patients [11, 13]. Difference may also exist between both sexes. Thus a personalized approach is required for every endograft procedure for ascending aorta lesions.

Our aim was to obtain an insight into normative values for the ascending aorta. The limitation of our study is the relatively low number of the studied population. In order to establish more solid normative tables, a larger population is needed. Besides we do not stratify our results by height and weight. Such normalization will be useful particularly in the pediatric population. Nevertheless, this study is a first step in establishing normative values as well as potential preoperative aortic characteristics in the perspective of future TCT of the ascending aorta. We think that ECGgated computed tomography is the most suitable and convenient approach for such a purpose.

\section{Conclusion}

Large inter-individual variations exist in measurements of the ascending aorta but with limited intra-individual variations during the cardiac cycle. Therefore a personalized design of transcatheter aortic devices for each patient might become necessary. ECG-gated MDCT could have a major role in the decision-making process and the treatment planning.

\section{References}

1. Dubost C, Allary M, Oeconomos N (1951) Aneurysm of the abdominal aorta treated by resection and graft. Arch Mal Coeur Vaiss 44:848-851
2. Dubost C, Allary M, Oeconomos N (1952) Resection of an aneurysm of the abdominal aorta: reestablishment of the continuity by a preserved human arterial graft, with result after five months. AMA Arch Surg 64:405-408
3. Creech O Jr (1966) Endoaneurysmorrhaphy and treatment of aortic aneurysm. Ann Surg 164:935946 
4. Parodi JC, Palmaz JC, Barone HD (1991) Transfemoral intraluminal graft implantation for abdominal aortic aneurysms. Ann Vasc Surg 5:491-499

5. Parker MV, O'Donnell SD, Chang AS, Johnson CA, Gillespie DL, Goff JM, Rasmussen TE, Rich NM (2005) What imaging studies are necessary for abdominal aortic endograft sizing? A prospective blinded study using conventional computed tomography, aortography, and three-dimensional computed tomography. J Vasc surg 41:199-205

6. Moon MC, Morales JP, Greenberg RK (2007) The aortic arch and ascending aorta: are they within the endovascular realm? Semin Vasc Surg 20:97-107

7. Huber CH, von Segesser LK (2006) Direct access valve replacement (DAVR) - are we entering a new era in cardiac surgery? Eur J Cardiothorac Surg 29:380-385

8. Ishimaru S (2004) Endografting of the aortic arch. J Endovasc Ther 11 Suppl 2:II62-71

9. Kasirajan K (2006) Thoracic endografts: procedural steps, technical pitfalls and how to avoid them. Semin Vasc Surg 19:3-10

10. Aronberg DJ, Glazer HS, Madsen K, Sagel SS (1984) Normal thoracic aortic diameters by computed tomography. J Comput Assist Tomogr 8:247-250

11. Pearce WH, Slaughter MS, LeMaire S, Salyapongse AN, Feinglass J, McCarthy WJ, Yao JS (1993) Aortic diameter as a function of age, gender, and body surface area. Surgery 114:691-697

12. Fitzgerald SW, Donaldson JS, Poznanski AK (1987) Pediatric thoracic aorta: normal measurements determined with CT. Radiology 165:667669

13. Hager A, Kaemmerer H, RappBerhhardt U, Blücher S, Rapp K, Bernhardt TM, Galanski M, Hess J (2002) Diameters of the thoracic aorta throughout life as measured with helical computed tomography. J Thorac Cardiovasc Surg 123:1060-1066

14. Rubin GD, Leung AN, Robertson VJ, Stark P (1998) Thoracic spiral CT: influence of subsecond gantry rotation on image quality. Radiology 208:771776
15. Qanadli SD, El Hajiam M, Mesurolle B, Lavisse L, Jourdan O, Randoux B, Chagnon S, Lacombe P (1999) Motion artifacts of the aorta simulating aortic dissection on spiral CT. J Comput Assist Tomogr 23:1-6

16. Duvernoy O, Coulden R, Ytterberg C (1995) Aortic motion: a potential pitfall in CT imaging of dissection in the ascending aorta. J Comput Assist Tomogr 19:569-572

17. Marten K, Funke M, Rummeny EJ, Engelke C (2005) Electrocardiographic assistance in multidetector CT of thoracic disorders. Clin Radiol 60:8-21

18. Roos JE, William JK, Weishaupt D, Lachat M, Marincek B, Hilfiker PR (2002) Thoracic aorta: motion artifact reduction with retrospective and prospective electrocardiography-assisted multi-detector row CT. Radiology 222:271-277

19. Schertler T, Glücker T, Wildermuth S, Jungius KP, Marincek B, Boehm T (2005) Comparison of retrospectively ECG-gated and nongated MDCT of the chest in an emergency setting regarding workflow, image quality, and diagnostic certainty. Emerg Radiol 12:19-29

20. O'Brien JP, Srichai MB, Hecht EM, Kim DC, Jacobs JE (2007) Anatomy of the heart at multidetector CT: what the radiologist needs to know. Radiographics 27:1569-1582

21. Horiguchi J, Kiguchi M, Fujioka C, Shen Y, Arie R, Sunasaka K, Ito K (2008) Radiation dose, image quality, stenosis mesurement, and CT densitometry using ECG-triggered coronary 64-MDCT angiography: a phantom study. AJR Am J Roentgenol 190:315320

22. van Prehn J, Vincken KL, Muhs BE, Barwegen GK, Bartels LW, Prokop M Moll FL, Verhagen HJ (2007) Toward endografting of the ascending aorta: insight into dynamics using dynamic cine-CTA. J Endovasc Ther 14:551-60

23. Ganten M, Krautter U, Hosch W, Hansmann J, von Tengg-Kobligk $\mathrm{H}$, Delorme S, Kauczor HU, Kauffmann GW, Bock M (2007) Age related change of human aortic distensibility: evaluation with ECG-gated CT. Eur Radiol 17:701-708

24. Poutanen T, Tikanoja T, Sairanen H, Jokinen E (2003) Normal aortic dimensions and flow in 168 children and young adults. Clin Physiol Funct Imaging 23:224-229

25. Smiseth OA, Stoylen A, Ihlen H (2004) Tissue Doppler imaging for the diagnosis of coronary artery disease. Curr Opin Cardiol 19:421-429
26. Van Dantzig JM (2006) Echocardiography in the emergency department. Semin Cardiothorac Vasc Anesth 10:79-81

27. Erbel R, Mohr-Kahaly S, Oelert H, Iversen S, Jakob H, Thelen M, Just M, Meyer J (1992) Diagnostic goals in aortic dissection. Value of transthoracic and transesophageal echocardiography. Herz 17:321-37

28. Appelbe AF, Walker PG, Yeoh JK, Bonitatibus A, Yoganathan AP, Martin RP (1993) Clinical significance and origin of artifacts in transesophageal echocardiography of the thoracic aorta. J Am Coll Cardiol 21:754-760

29. Clarkson PM, Brandt PW (1985) Aortic diameters in infants and young children: normative angiographic data. Pediatr Cardiol 6:3-6

30. Trivedi KR, Pinzon JL, McCrindle BW, Burrows PE, Freedom RM, Benson LN (2002) Cineangiographic aortic dimensions in normal children. Cardiol Young 12:339-344

31. Finet $\mathrm{G}$, Masquet $\mathrm{C}$, Eifferman A, Funck F, Lefèvre T, Marco J, Amiel M, Beaune J, Liénard J (1996) Can we optimize our angiographic views every time? Qualitative and quantitative evaluation of a new functionality. Invest Radiol 31:523-531

32. Lorenz $\mathrm{CH}$ (2000) The range of normal values of cardiovascular structures in infants, children, and adolescents measured by magnetic resonance imaging. Pediatr Cardiol 21:37-46

33. Kim YC, Nielsen JF, Nayak KS (2008) Automatic correction of echo-planar imaging (EPI) ghosting artifacts in realtime interactive cardiac MRI using sensitivity encoding. J Magn Reson Imaging 27:239-245

34. Tenforde TS (2005) Magnetically induced electric fields and currents in the circulatory system. Prog Biophys Mol Biol 87:279-288

35. Wyers MC, Fillinger MF,

Schermerhorn ML, Powell RJ, Rzucidio EM, Walsh DB, Zwolak RM, Cronenwett JL (2003) Endovascular repair of abdominal aortic aneurysm without preoperative arteriography. J Vasc Surg 38:730-738

36. von Ludinghausen M (2003) The clinical anatomy of coronary arteries. Adv Anat Embryol Cell Biol 167:III-VIII, $1-111$

37. Huber $\mathrm{CH}$, Tozzi P, Corno AF, Marty B, Ruchat P, Gersbach P, Nasratulla M, von Segesser LK (2004) Do valved stents compromise coronary flow? Eur J Cardiothorac Surg 25:754-759 